\title{
METHODS IN CLINICAL PHARMACOLOGY
}

\section{Methodological quality of antimalarial randomized controlled trials during pregnancy and its impact on the risk of low birth weight}

Correspondence Dr Anick Bérard, Research Chair on Medications, Pregnancy and Lactation, Faculty of Pharmacy, University of Montreal and Director of Research Unit on Medications and Pregnancy, Research Center CHU Ste-Justine 3175, Côte-Sainte-Catherine, Montréal (Québec), H3T 1C5, poste 4363, Canada. Tel.: +1 (514) 345 4931; Fax: +1 (514) 343 6120; E-mail: anick.berard@umontreal.ca

Received 8 December 2015; revised 28 April 2016; accepted 30 April 2016

Flory T. Muanda ${ }^{1,2}$ and Anick Bérard ${ }^{1,2}$

${ }^{1}$ Faculty of PharmacyUniversity of Montreal, 2900 Édouard Montpetit, Montréal, Québec, Canada, H3T 1J4 and ${ }^{2}$ Research Center, CHU Sainte-Justine, 3175, Côte-Sainte-Catherine, Montréal, Québec, Canada, H3T 1C5

Keywords meta-regression, randomized controlled trials, risk of bias

AlM

To describe biases in antimalarial randomized controlled trials (RCTs) during pregnancy and their influence on antimalarial drug efficacy to reduce the risk of low birth weight (LBW).

\section{METHODS}

RCT characteristics and results were retrieved from a previous systematic review on the efficacy of antimalarials. The Cochrane risk of bias assessment was used to investigate source of biases in each RCT. The quality of RCT reporting published after the introduction of the CONSORT statement in medical literature in 1996 were compared to those published before 1996. A metaregression analysis was performed to examine the impact of bias on the efficacy of antimalarials to reduce LBW after controlling for the time period prior to 1996.

\section{RESULTS}

Twenty out of 25 RCTs (80\%) had a high risk of bias. The proportion of RCTs having a low risk of bias was higher in manuscripts published after the introduction of CONSORT compared to those published before 1996 for sequence generation $(P=0.04)$ and allocation concealment $(P=0.04)$. Heterogeneity between RCTs was associated with an overestimation of the efficacy of antimalarial drugs in reducing LBW in RCTs with inadequate methods for randomization, allocation concealment or not being free of other bias.

\section{CONCLUSION}

Antimalarial RCTs during pregnancy are poorly reported but may be improved by using the CONSORT statement. After taking into account the time period before 1996, we found that biases had an impact on the efficacy of antimalarials to reduce the risk of LBW. 
WHAT IS ALREADY KNOWN ABOUT THIS SUBJECT

- Previous studies showed poor reporting of the methodology used in RCTs and suggested that the influence of biases in RCTs on treatment effect estimates may vary according to types of outcomes (subjective or objective outcome).

WHAT THIS STUDY ADDS

- Methodological quality of antimalarial RCTs during pregnancy is poor but is improved with the use of the CONSORT statement.

- Biases had a statistically significant impact on the efficacy of antimalarial drugs in reducing LBW in RCTs with inadequate methods for randomization, allocation concealment or not being free of other bias.

- Researchers should assess the influence of methodological flaws on the efficacy of antimalarials in meta-analyses and encourage the use of the CONSORT statement.

\section{Introduction}

Randomized controlled trials (RCTs) provide the best evidence for clinical and policy decision making about medical interventions. However, bias cannot be ruled out completely [1]. Many tools have been developed to assess the quality of RCTs for systematic reviews [2]. These instruments include quality scales and checklists, which are used interchangeably [3].The Consolidated Standards of Reporting Trials (CONSORT) statement was implemented in 1996 to help authors improve the quality of RCT reporting [4]. Readers, peer reviewers and editors can also use the CONSORT statement to critically appraise and interpret reporting of RCTs, even though it was not originally designed for quality assessment [5]. Studies have shown that the quality of RCT reporting has improved since the introduction of this checklist in some journal guidelines [5-7]. The Cochrane collaboration group developed the Cochrane risk of bias tool, a new instrument, which is neither a checklist nor a scale, in order to better assess the methodological quality of RCTs [2]. It is a domain-based evaluation in which critical assessments are made separately for different domains. Despite efforts to minimize biases in RCTs, the quality of reporting remains suboptimal leading to potential incorrect results [8].

Meta-epidemiological studies evaluate the influence of study design characteristics on the results of RCTs [9]. Evidence based on the largest meta-epidemiological study suggested that RCTs with unclear or inadequate methods of randomization, methods of allocation concealment of the treatment and unclear or lack of blinding tended to overestimate the treatment efficacy compared to those using proper methods of randomization, allocation concealment and blinding [10]. Nevertheless, the influence of these biases on the treatment effect may vary according to the outcome type (i.e. subjective $v s$. objective) $[11,12]$. Hence, current evidence showed that if the outcome is subjective, treatment efficacy is overestimated by $15 \%$ in RCTs with unclear or inadequate allocation concealment, $17 \%$ in RCTs with unclear or inadequate random allocation of treatment, and $22 \%$ if double blinding is lacking [10]. Conversely, there is no clear evidence of overestimation of treatment efficacy in RCTs assessing objective outcomes, even with inadequate method of randomization or allocation concealment [10].

It has been shown that the efficacy of antimalarial drugs in preventing adverse pregnancy outcomes may vary according to drug resistance level, gravidity and HIV status [13, 14]. Yet, the impact of the study design characteristics of antimalarial RCTs on their results have not been reported in the literature. Also, whether the introduction of the CONSORT statement improved reporting of antimalarial RCTs is still unknown. Therefore, our objectives were (1) to describe biases that might arise from antimalarial RCTs in pregnant women, (2) to evaluate whether the introduction of the CONSORT statement in 1996 influenced the quality of RCT reporting, and (3) to examine the impact of biases in RCTs on antimalarial drugs efficacy used for malaria prevention during pregnancy in preventing low birth weight (LBW), after taking into account the time period prior to the introduction of the CONSORT statement in 1996. We used a recent systematic review looking at the efficacy of antimalarial drugs for malaria prevention during pregnancy in reducing the risk of LBW as an illustrative case study [13].

\section{Method}

\section{Study selection}

RCTs included in the analysis were identified from a large systematic review described in detail elsewhere [13]. Briefly, to be included in this review, studies had to: (1) evaluate antimalarial drugs for prevention of malaria during pregnancy,(2) report data on LBW (LBW was defined as a live born with a birth weight $<2,500 \mathrm{~g}$ regardless of gestational age), (3) contain data on pregnant women exposed to any type of antimalarial used for malaria prevention and a comparator group (no use of antimalarial drugs, placebo or other types of antimalarials), (4) to report a Plasmodium Falciparum infection, (5) be RCTs or quasi-RCTs, (6) be published in French or English, and (7) be identified in PubMed, EMBASE or in the Cochrane Central Register of Controlled Trials from their inception to November 21, 2014.

\section{Data extraction and risk of bias assessment}

For each RCT included in the review, we extracted the risk of bias assessment and risk ratio (RR) alongside their 95\% confidence interval (CI).

The Cochrane risk of bias tool was used to assess the methodological quality of the RCTs [2]. Briefly, it is divided into two parts and covers six specific domains that are potential sources of bias in RCTs: (1) sequence of generation, (2) allocation concealment, (3) blinding of participants, personnel and outcome assessors, (4) incomplete outcome data, (5) selective reporting description, and (6) other sources of bias [2]. For 
each assessed domain, the first part provides a summary of the methods used in RCTs and the second part assigns a judgement of low, unclear or high risk of bias [2].

Since the creation of the CONSORT in 1996, studies showed that the quality of RCT reporting has improved [6-8], as it was introduced within journal guidelines. An improvement in RCT reporting has also been shown in manuscripts published after the introduction of this checklist in the medical literature [15]. Therefore, we extracted the proportion of RCTs with low risk of bias (good internal validity) for each domain according to the Cochrane risk of bias tool for RCTs included in our review published before 1996 (the pre-CONSORT period) and after 1996 (post-CONSORT statement).

\section{Data synthesis}

Description of risk of bias in antimalarial RCTs and influence of the CONSORT statement in the quality of RCT reporting using the Cochrane risk of bias tool. We assessed the risk of bias for all RCTs included in this review and for each assessed domain using the Cochrane risk of bias tool. We carried out a comparative study to investigate the influence of the introduction of the CONSORT statement in the medical literature in 1996. We compared the proportion of RCTs with low risk of bias (good internal validity) published before 1996 (pre-CONSORT period) that published after 1996 (post-CONSORT period) for each domain using a onesided Fisher exact test $(P$-value $<0.05$ were considered significant) [16]. All statistical analyses were performed with SAS software (version 9.3; SAS Institute Inc., Cary, NC, USA).

Impact of bias on the efficacy of antimalarial drugs in reducing $L B W$ risk. For each domain assessed by the Cochrane risk of bias tool, we performed a meta-analysis (random-effects model) in each sub-group classified as low, high and unclear risk of bias. When the point estimates for the subgroup including RCTs with high or unclear risk of bias were different from the subgroup including RCTs with low risk of bias, we performed a meta-regression analysis to quantify the risk difference with 95\% CI [17]. This method is an extension to subgroup analyses that allows researchers to assess simultaneously the influence of the study characteristics that may affect the efficacy of a treatment [18]. Meta-regression is similar to simple regression except that it uses weighted data from multiple RCTs rather than individual patient data from a single study [2]. The dependent variable in meta-regression is the effect estimate, and the explanatory variables (potential effect modifiers or covariates) are any characteristics of the study that might influence the effect estimate. For categorical explanatory variables, regression coefficients estimate how the intervention effect in each subgroup differs from a nominated reference subgroup [2]. Each regression coefficient's $P$-value indicates whether this difference is statistically significant.

In our study, the dependent variable was the effect estimate (log RR) from each RCT included in our review. The judgement of risk of bias assigned to RCTs was considered a potential effect modifier of the efficacy of antimalarial drugs in reducing LBW risk, and thus was included in the model as an independent variable.
Given that three different levels of judgement could be assigned to an RCT for each assessed domain, a dummy variable was created and the level of low risk of bias was used as the reference subgroup. Both high and unclear risks of bias were included in the model as covariates. We also included in our model the time period prior to the introduction of the CONSORT statement in 1996 as a potential confounder of the association between bias and the efficacy of the antimalarial drugs. A random effect meta-regression was used to assess the influence of risk of bias on the efficacy of the antimalarial drugs in reducing LBW risk after controlling for the time period prior to 1996 [17]. The following expression was used in our model [19]: $\log \mathrm{RR}=a+\beta_{1} x_{1}+\beta_{2} x_{2}+\beta_{3} x_{3}+u_{i}+e_{i}$ where $a$ is a constant, $u_{i}$ is a normal error term with known study variance $\left(\operatorname{se}(\beta)^{2}\right)$, that might vary across RCTs, and $e_{i}$ is a normal error term with a between study variance $\left(\tau^{2}\right)$ to be estimated and assumed to be equal across RCTs [19].

The $\beta_{1}$ regression coefficient estimated how the efficacy of the antimalarial drugs in reducing LBW risk in each RCT with a high risk of bias differed from the reference subgroup (RCTs with low risk of bias). The $\beta_{2}$ regression coefficient estimated how the efficacy of the antimalarial drugs in reducing the risk of LBW in each RCT with unclear risk of bias differed from the reference subgroup (RCTs with low risk of bias). The $\beta_{3}$ regression coefficient estimated how the efficacy of the antimalarial drugs in reducing the risk of LBW in each RCT published prior to 1996 differed from the reference subgroup (RCTs published after 1996).

The restricted maximum likelihood (RELM) algorithm was used in our model to estimate the between study variance $\left(\tau^{2}\right)$ and then regression coefficients for RCTs with high risk of bias and unclear risk of bias were calculated using each RCT assigned weight, also given by the formula [20]:

$$
\mathrm{W}_{\mathrm{i}}=\frac{1}{\operatorname{se}(\beta \mathrm{s})^{2}+\tau^{2}}
$$

When $\beta_{1}$ or $\beta_{2}$ was $<0$, the result indicated that RCTs with high or unclear risk of bias were associated with an overestimation of the efficacy of the antimalarial drugs. Conversely when $\beta_{1}$ or $\beta_{2}$ was $>0$, the result indicated an underestimation of the efficacy of the antimalarial drugs. When $\beta_{3}<0$, the result indicated that antimalarial drugs were more effective at reducing the risk of LBW in RCTs published prior to 1996 compared to those published after 1996. In contrast, when $\beta_{3}>0$, the result indicated that antimalarial drugs were less effective at reducing the risk of LBW in RCTs published prior to 1996.

All analyses were performed using STATA version 12.1 [19].

\section{Results}

\section{Description of biases in antimalarial RCTs according to the Cochrane risk of bias tool}

A total of 25 RCTs were included in the review. The majority of these studies (96\%) were conducted in Africa and only one was carried out in Asia. The median year of publication was 2008 (IQR, 2004-2009). Most RCTs had overall high $(80 \%)$ or unclear $(12 \%)$ risk of bias assessments. Only two studies out of $25(8 \%)$ were classified as having low risk of 
bias. The methodological quality of RCTs included in this review is described for each domain in Table 1 . Selective reporting $(60 \%)$, sequence generation of randomization (44\%) and allocation concealment (44\%) were the domains most frequently rated as low risk of bias. Other source of bias (56\%) was more likely assessed with high risk of bias, and selective reporting (40\%) with the unclear risk of bias. Compared to studies published before 1996, the proportion of RCTs with a low risk of bias was significantly higher in studies published after the introduction of the CONSORT statement for sequence generation $(P=0.04)$ and allocation concealment $(P=0.04)$ (Table 1$)$. Overall, there was no significant differences in proportion of RCTs with low risk of bias for all domains assessed between the two period $(P=0.63)$ (Table 1$)$.

\section{Impact of bias on the efficacy of antimalarial} drugs in reducing the risk of $L B W$ according to the Cochrane risk of bias domain: subgroup analysis and meta-regression after controlling for the time period prior to introduction of the CONSORTstatement in 1996

A subgroup analysis and a meta-regression of placebocontrolled RCTs were performed to assess the impact of biases on the efficacy of antimalarial drugs in reducing LBW risk after taking into account the time period prior to introduction of the CONSORT statement in 1996.

\section{Sequence generation}

The subgroup analysis resulted in a point estimate of 0.48 (95\% CI: $0.18,1.26)$ for RCTs with high risk of bias, 0.72 (95\% CI: $0.43,1.22)$ for unclear risk of bias and $0.97(95 \%$ CI: $0.80,1.17$ ) for RCTs with low risk of bias (Supporting Information Figure S1). Our meta-regression analysis showed a statistically significant difference when we compared RCTs with high risk of bias (adjusted regression coefficient $=-1.02$, $95 \%$ CI: $-2.06,-0.001, P=0.05)$ to those with low risk of bias (reference group) (Figure 1). The difference was not significant when RCTs with unclear risk of bias (adjusted regression coefficient $=-0.59,95 \% \mathrm{CI}:-1.52,0.33, P=0.17$ ) were compared with those with low risk of bias (reference group) (Figure 1). Our meta-regression also did not show a statistically significant difference when RCTs published before 1996 were compared with those published after this period (adjusted regression coefficient $=0.69,95 \% \mathrm{CI}:-0.21,1.59, P=0.11$ ).

\section{Allocation concealment}

The subgroup analysis resulted in a point estimate of 0.48 (95\% CI: $0.22,1.02)$ for RCTs with high risk of bias, 0.81 (95\% CI: $0.45,1.48)$ for unclear risk of bias and $0.97(95 \%$ CI: $0.80,1.17$ ) for RCTs with low risk of bias (Supporting Information Figure S1). Our meta-regression analysis found a statistically significant difference when we compared RCTs with high risk of bias (adjusted regression coefficient $=-1.22$,

\section{Table 1}

Risk of bias assessment by domains and comparison of quality report of trials published before and after the introduction of the CONSORT statement

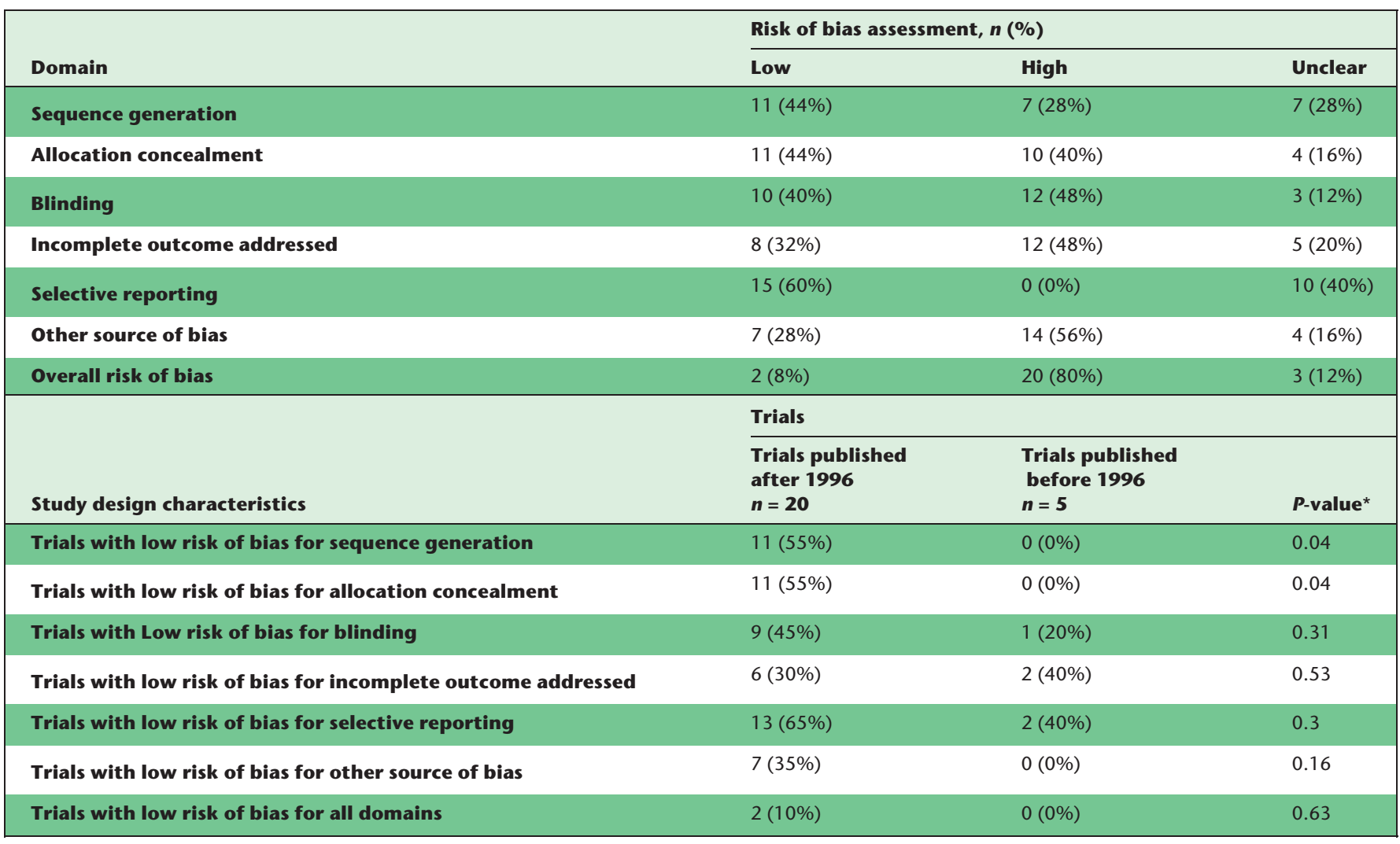

* $P$-value $<0.05$ were considered significant 


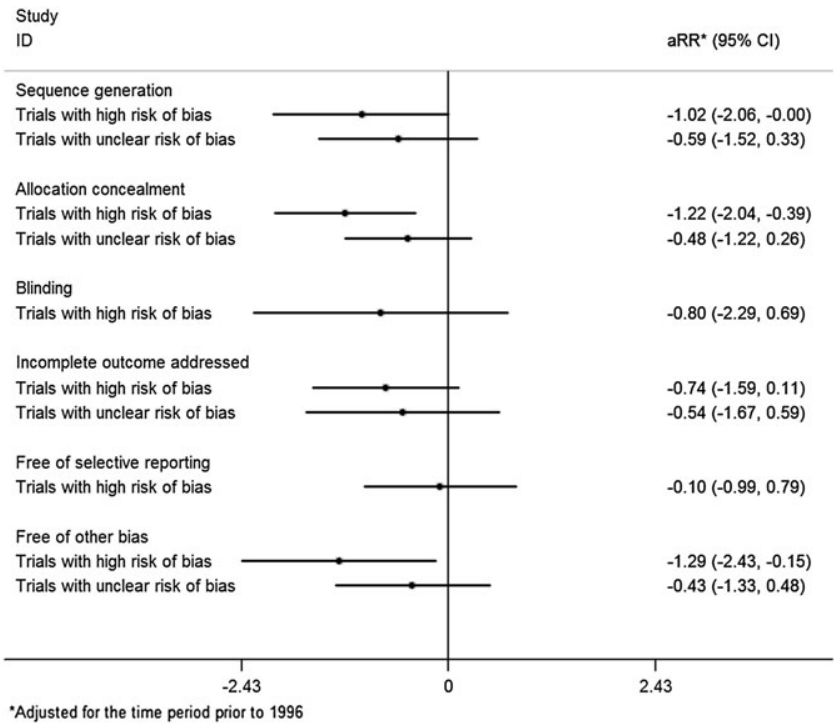

Figure 1

Forest plot depicting risk difference of the treatment effect estimates of antimalarial drugs on the risk of LBW stratified by Cochrane risk of bias domain

95\% CI: $-2.04,-0.39, P=0.01)$ with those with low risk of bias (Figure 1). The difference was not significant when RCTs with unclear risk of bias (adjusted regression coefficient $=-0.48$, 95\% CI: $-1.22,0.26, P=0.17$ ) were compared with those with low risk of bias (reference group). (Figure 1). We found a statistically significant difference when RCTs published before 1996 were compared with those published after this period (adjusted regression coefficient $=0.94,95 \% \mathrm{CI}: 0.17,1.70, P=0.02$ ).

\section{Blinding}

The RR was 0.62 (95\% CI: $0.31,1.22)$ for RCTs with high risk of bias and 0.76 (95\% CI: 0.53, 1.08) for RCTs with low risk of bias (Supporting Information Figure S1). Our meta-regression analysis showed no risk difference between the two groups (adjusted regression coefficient $=-0.80,95 \%$ CI: -2.29 , $0.69, P=0.25$ ) (Figure 1). Similar results were found when RCTs published before 1996 were compared with those published after this period (adjusted regression coefficient $=0.72$, 95\% CI: $-0.66,2.09, P=0.26)$.

\section{Incomplete outcome addressed}

The RR was 0.56 (95\% CI: $0.34,0.91)$ for RCTs with high risk of bias; 0.63 (95\% CI: $0.39,1.00)$ for RCTs with unclear risk of bias; 1.09 (95\% CI: 0.88, 1.34) for RCTs with low risk of bias (Supporting Information Figure S1). Our meta-regression analysis also did not show any statistically significant difference when we compared RCTs with high risk of bias (adjusted regression coefficient $=-0.74,95 \% \mathrm{CI}:-1.59,0.11, P=0.08$ ) or unclear risk of bias (adjusted regression coefficient $=-0.54,95 \% \mathrm{CI}:-1.67$, $0.59, P=0.29)$ to those with low risk of bias (reference group) (Figure 1)

Similar results were also found when RCTs published before 1996 were compared with those published after this period (adjusted regression coefficient $=0.28$, 95\% CI: $-0.56,1.13, P=0.44)$.

\section{Free of selective reporting}

The RR was 0.68 (95\% CI: $0.39,1.16)$ for RCTs with unclear risk of bias and 0.80 (95\% CI: $0.59,1.09)$ for RCTs with low risk of bias (Supporting Information Figure S1). Our meta-regression analysis also showed no statistically significant difference between the two groups (adjusted regression coefficient = $-0.10,95 \%$ CI: $-0.99,0.79, P=0.80$ ) (Figure 1). Similar results were also found when RCTs published before 1996 were compared with those published after this period (adjusted regression coefficient $=0.14,95 \% \mathrm{CI}:-0.78,1.06, P=0.73$ ) .

\section{Free of other bias}

The RR was 0.60 (95\% CI: $0.32,1.12)$ for RCTs with high risk of bias, 0.63 (95\% CI: $0.39,1.00)$ for unclear risk of bias and 0.97 (95\% CI: 0.80, 1.17) for RCTs with low risk of bias (Supporting Information Figure S1). Our meta-regression analysis found a statistically significant difference when we compared RCTs with high risk of bias (adjusted regression coefficient $=-1.29$, 95\% CI: $-2.43,-0.15, P=0.03)$ with those with low risk of bias (reference group) (Figure 1). The difference was not significant when RCTs with unclear risk of bias (adjusted regression coefficient $=-0.43,95 \% \mathrm{CI}:-1.33,0.48, P=0.29)$ were compared with those with low risk of bias (Figure 1).

Our meta-regression analysis found a significant difference when RCTs published before 1996 were compared with those published after this period (adjusted regression coefficient $=1.19,95 \%$ CI: $0.05,2.33, P=0.04$ ).

\section{Discussion}

Our study showed that most antimalarial RCTs conducted during pregnancy had overall high risk or unclear risk of bias. Previous studies using the Cochrane risk of bias tool found similar results with paediatrics and adult RCTs [21, 22]. Our study also reported a high risk or unclear risk of bias for many assessed domains. This result was consistent with a previous study describing malaria trials in Africa [23]. Our study found that the proportion of RCTs with low risk of bias published after the CONSORT statement implementation in 1996 was significantly higher than those published before this period regarding sequence generation of randomization and allocation concealment. This may suggest a better reporting of RCTs after the introduction of this statement in the medical literature. Similarly a study assessing the quality of reporting of RCTs in ovary polycystic syndrome also showed that the post-CONSORT implementation was associated with a better reporting of sequence generation $(P<0.01)$ and allocation concealment $(P<0.01)$ compared to the pre-CONSORT period [15]. Despite the implementation of the CONSORT statement, our study did not show significant increase in the proportion of RCTs having a low risk of bias for all domains assessed. This finding was consistent with a Cochrane review suggesting that RCT reporting remained suboptimal despite relative improvements after the endorsement of the CONSORT by journals [24]. However, the methodological quality of RCTs may have been underestimated in this study due to poor or unclear RCT reports (which does not necessarily mean that the trial was not well conducted) [25]. A recent study that assessed the reliability of the methodological 
quality of RCTs based on published reports of RCTs into cancer showed a significant increase in the proportion of RCTs with low risk of bias for all individual domains when additional information was provided by the authors or after reviewing the RCT protocols [26].

Our study found that RCTs with inadequate methods of randomization and allocation concealment were associated with an overestimation of the efficacy of antimalarial drugs in reducing the risk of LBW after controlling for the time period before the introduction of the CONSORT statement in 1996. This result is consistent with the largest meta-epidemiological study that showed overall an overestimation of treatment efficacy in RCTs with an inadequate method of randomization and allocation concealment, and a greater effect when the outcome was subjectively assessed [10]. This finding was also similar to previous studies that showed an exaggeration of the treatment benefit in trials with low methodological quality for randomization and allocation concealment $[27,28]$. RCTs with inadequate methods addressing other types of bias, such as baseline imbalance, were also associated with an overestimation of the efficacy of antimalarial drugs in reducing the risk of LBW in our study. This result highlights the need to compare the distribution of baseline covariates between treatment groups in RCTs, especially when randomization or allocation concealment methods have been compromised in the trial. Moreover, other potential sources of bias in RCTs, such as the use of inadequate or unclear methods for dealing with missing data, inadequate method to ensure blinding and selective outcome reporting, showed a trend towards an overestimation of the efficacy of antimalarial drugs in reducing the risk of LBW in our study. However, no regression coefficients have reached a statistical significance given the small number of studies including in the meta-analysis. These findings are consistent with a recent meta-epidemiological study conducted on a sample of paediatric RCTs that found a similar trend [21]. Our study also showed that antimalarial drugs appear to be less effective at reducing the risk of LBW in RCTs published before 1996 compared to those published after this period. A potential explanation for this finding is the prevalence of antimalarial drug resistance in those studies that were, on average, $20.32 \%$ (median rate of resistance: $21.45 \%$, interquartile range (IQR), 14.7-25.95\%), which was above the cut-off recommended (10\%) by the World Health Organization (WHO) for policy change in malaria areas [13].

To our knowledge, this is the first study to describe the risk of bias in antimalarial RCTs conducted during pregnancy and that assessed the influence of the CONSORT statement implementation on the quality of reporting of antimalarial RCTs using the Cochrane risk of bias tool. Our study is also the first to investigate the association between the risk of bias in RCTs included in a meta-analysis and the efficacy of antimalarial drugs on LBW, an objectively measured outcome. However, our study does include potential limitations.

First, we were unable to get additional information from the authors and not all RCT protocols were available in the literature. Therefore, the proportion of RCTs with low risk of bias may have been underestimated in this study as risk of bias assessment was solely based on publications.

Second, we assessed the influence of the CONSORT statement on quality of reporting of antimalarial RCTs using the period after its implementation as a proxy of use. It would have been more interesting to compare CONSORT endorsers' and non-endorsers' journals. However the limited number of studies included in our review precluded this analysis.

Finally, although LBW is classified as an objectively measured outcome [10], our study showed that biases in RCTs were associated with an overestimation of the efficacy of antimalarial drugs in reducing this adverse pregnancy outcome. A potential explanation for this finding was related to the methods used for weighing the babies which were not standardized across all the RCTs. Given that measures of LBW were different across the studies, the assessment of this outcome was more likely to be subjective across these RCTs.

\section{Conclusion}

In summary, most of antimalarial RCTs during pregnancy had a high or unclear risk of bias. The CONSORT statement implementation was associated with an improvement in RCT reporting regarding methods for randomization and allocation concealment. However, the overall proportion of RCTs with low risk of bias remained low but may also be underestimated in this study. After controlling for the time period prior to 1996, the risk of bias in RCTs was associated with an overestimation of the efficacy of antimalarial drugs in reducing LBW in RCTs with inadequate methods for randomization, inadequate methods for allocation concealment or not being free of other bias. These results highlight the need to promote the CONSORT statement use to report antimalarial RCTs and to investigate the impact of methodological heterogeneity on the efficacy of malaria treatment when performing a meta-analysis. Further studies are needed to confirm this finding.

\section{Competing Interests}

Both authors have completed the Unified Competing Interest form at www.icmje.org/coi_disclosure.pdf (available on request from the corresponding author) and declare: $\mathrm{AB}$ is a consultant for plaintiffs in the litigation involving antidepressants and birth defects. FTM reports no support from any organization for the submitted work; no financial relationships with any organizations that might have an interest in the submitted work in the previous 3 years; no other relationships or activities that could appear to have influenced the submitted work.

This research was funded by the Réseau Québécois de Recherche sur l'Usage des Médicaments (RQRUM). Flory Tsobo Muanda is the recipient of the Sainte-Justine Hospital Foundation and the Foundation of Stars scholarship from the Faculty of Pharmacy of the University of Montreal and is scholarship holder of the Canadian Network for Advanced Interdisciplinary Methods for comparative effectiveness research (CAN-AIM). Anick Berrard is recipient of a career award from the FRSQ and is on the endowment Research Chair of the Famille Louis-Boivin on Medications, Pregnancy and Lactation at the Faculty of Pharmacy of the University of Montreal. We would like to thank Dr Lucie Blais, Dr Sylvie Perrault, Dr Fabiano Santos, Ms Odile Sheehy, Ms Takoua Boukris, and Sonia Chabaane, who assess the risk of bias for each of the studies included in the review. 


\section{Contributors}

Dr Flory T. Muanda and Professor Anick Bérard participated in the design, data collection, analysis and interpretation of the data. This paper was principally drafted by Dr Flory T. Muanda and was critically reviewed and subsequently approved by Professor Anick Bérard in its final form.

\section{References}

1 Gluud LL. Bias in clinical intervention research. Am J Epidemiol 2006; 163: 493-501.

2 Higgins JP, Green S. Cochrane Handbook for Systematic Reviews of Interventions 4.2.5 (updated May 2005). London: Cochrane, 2005.

3 Juni P, Altman DG, Egger M. Systematic reviews in health care: Assessing the quality of controlled clinical trials. BMJ (Clinical research ed) 2001; 323: 42-6.

4 Begg C, Cho M, Eastwood S, Horton R, Moher D, Olkin I, et al. Improving the quality of reporting of randomized controlled trials. The CONSORT statement. JAMA 1996; 276: 637-9.

5 Moher D, Hopewell S, Schulz KF, Montori V, Gotzsche PC, Devereaux PJ, et al. CONSORT 2010 explanation and elaboration: updated guidelines for reporting parallel group randomised trials. BMJ (Clinical research ed).2010; 340: c869.

6 Hopewell S, Dutton S, Yu LM, Chan AW, Altman DG. The quality of reports of randomised trials in 2000 and 2006: comparative study of articles indexed in PubMed. BMJ (Clinical research ed).2010; 340: c723.

7 Egger M, Juni P, Bartlett C. Value of flow diagrams in reports of randomized controlled trials. JAMA 2001; 285: 1996-9.

8 Plint AC, Moher D, Morrison A, Schulz K, Altman DG, Hill C, et al. Does the CONSORT checklist improve the quality of reports of randomised controlled trials? A systematic review. Med J Aust 2006; 185: 263-7.

9 Naylor CD. Meta-analysis and the meta-epidemiology of clinical research. BMJ (Clinical research ed) 1997; 315: 617-9.

10 Savovic J, Jones HE, Altman DG, Harris RJ, Juni P, Pildal J, et al. Influence of reported study design characteristics on intervention effect estimates from randomized, controlled trials. Ann Intern Med 157: 429-38.

11 Wood L, Egger M, Gluud LL, Schulz KF, Juni P, Altman DG, et al. Empirical evidence of bias in treatment effect estimates in controlled trials with different interventions and outcomes: meta-epidemiological study. BMJ (Clinical research ed).2008; 336: 601-5.

12 Balk EM, Bonis PA, Moskowitz H, Schmid CH, Ioannidis JP, Wang $\mathrm{C}$, et al. Correlation of quality measures with estimates of treatment effect in meta-analyses of randomized controlled trials. JAMA 2002; 287: 2973-82.

13 Muanda FT, Chaabane S, Boukhris T, Santos F, Sheehy O, Perreault $S$, et al. Antimalarial drugs for preventing malaria during pregnancy and the risk of low birth weight: a systematic review and meta-analysis of randomized and quasi-randomized trials. BMC Med 2015; 13: 193.

14 Kayentao K, Garner P, van Eijk AM, Naidoo I, Roper C, Mulokozi A, et al. Intermittent preventive therapy for malaria during pregnancy using 2 vs 3 or more doses of sulfadoxine- pyrimethamine and risk of low birth weight in Africa: systematic review and meta-analysis. JAMA 2013; 309: 594-604.

15 Partsinevelou A, Zintzaras E. Quality of reporting of randomized controlled trials in polycystic ovary syndrome. Trials 2009; 10: 106.

16 Lydersen S, Fagerland MW, Laake P. Recommended tests for association in $2 \times 2$ tables. Stat Med 2009; 28: 1159-75.

17 Thompson SG. Why and how sources of heterogeneity should be investigated. In: Systematic Reviews in Health Care, eds Egger M, Smith GD, Altman DG. London: BMJ Publishing Group, 2008; 157-75.

18 Baker WL, White CM, Cappelleri JC, Kluger J, Coleman CI. Understanding heterogeneity in meta-analysis: the role of metaregression. Int J Clin Pract 2009; 63: 1426-34.

19 Sterne JAC, Bradburn MJ, Egger M. Meta-analysis in Stata ${ }^{\mathrm{TM}}$. In: Systematic Reviews in Health Care, eds Egger M, Smith GD, Altman DG. London: BMJ Publishing Group, 2008; 347-69.

20 Harbord R, Higgins J. Meta-regression in Stata. Stata J 2008; 8: 493-519.

21 Hartling L, Hamm MP, Fernandes RM, Dryden DM, Vandermeer B. Quantifying bias in randomized controlled trials in child health: a meta-epidemiological study. PLoS One 2014; 9: e88008

22 Hartling L, Hamm MP, Milne A, Vandermeer B, Santaguida PL, Ansari $\mathrm{M}$, et al. Testing the risk of bias tool showed low reliability between individual reviewers and across consensus assessments of reviewer pairs. J Clin Epidemiol 2013; 66: 973-81.

23 Lutje V, Gerritsen A, Siegfried N. Randomized controlled trials of malaria intervention trials in Africa, 1948 to 2007: a descriptive analysis. Malar J 2011; 10: 61.

24 Turner L, Shamseer L, Altman DG, Schulz KF, Moher D. Does use of the CONSORT Statement impact the completeness of reporting of randomised controlled trials published in medical journals? A Cochrane review. Sys Rev 2012; 1: 60.

25 Soares HP, Daniels S, Kumar A, Clarke M, Scott C, Swann S, et al. Bad reporting does not mean bad methods for randomised trials: observational study of randomised controlled trials performed by the Radiation Therapy Oncology Group. BMJ (Clinical research ed) 2004 ; 328: 22-4.

26 Vale CL, Tierney JF, Burdett S. Can trial quality be reliably assessed from published reports of cancer trials: evaluation of risk of bias assessments in systematic reviews. BMJ (Clinical research ed). 2013; 346: f1798.

27 Moher D, Pham B, Jones A, Cook DJ, Jadad AR, Moher M, et al. Does quality of reports of randomised trials affect estimates of intervention efficacy reported in meta-analyses? Lancet 1998; 352: 609-13.

28 Pildal J, Hrobjartsson A, Jorgensen KJ, Hilden J, Altman DG, Gotzsche PC. Impact of allocation concealment on conclusions drawn from meta-analyses of randomized trials. Int J Epidemiol 2007; 36: 847-57.

\section{Supporting Information}

Additional Supporting Information may be found in the online version of this article at the publisher's web-site:

http://onlinelibrary.wiley.com/doi/10.1111/bcp.12998/suppinfo.

Figure S1a All combined antimalarial drugs as compared to no use of antimalarial drugs and risk of LBW stratified by sequence generation of randomization 
Figure $\mathbf{S 1 b}$ All combined antimalarial drugs as compared to no use of antimalarial drugs and risk of LBW stratified by allocation concealment

Figure S1c All combined antimalarial drugs as compared to no use of antimalarial drugs and risk of LBW stratified by blinding

Figure S1d All combined antimalarial drugs as compared to no use of antimalarial drugs and risk of LBW stratified by incomplete outcome addressed
Figure S1e All combined antimalarial drugs as compared to no use of antimalarial drugs and risk of LBW stratified by free of selective reporting domain

Figure S1f All combined antimalarial drugs as compared to no use of antimalarial drugs and risk of LBW stratified by free of other bias domain 